\title{
VAC_22 - Development of COVID-19 vaccine: the Bio-Manguinhos initiative
}

Gabriela dos Santos Esteves ${ }^{1 *}$; Patrícia Cristina da Costa Neves ${ }^{1}$; Ana Paula Dinis Ano Bom'; Sheila Maria Barbosa de Lima ${ }^{1}$; Rodrigo Muller ${ }^{1}$; Janaina Figueira Mansur ${ }^{1}$; Rodrigo Nunes Rodrigues da Silva ${ }^{1}$; Sotiris Missailidis ${ }^{1}$; Juliana Gil Melgaço'; Waleska Dias Schwarcz ${ }^{1}$.

${ }^{1}$ Fiocruz/Bio-Manguinhos.

Introduction: Vaccines are the most effective strategy to prevent infectious diseases. COVID-19 is caused by the Sars-CoV-2 virus and it was declared a pandemic disease in March of 2020. Since then, research groups all over the world are working to develop and produce a safe and effective vaccine to avoid deaths and the health system collapse. Now the world is facing the limited amount of vaccine supply, so it is important to have a vaccine produced in Brazil in an attempt to supply the country needs and guarantee future demand following the pandemic period. The Technological Development Division of Bio-Manguinhos/Fiocruz is working on two different strategies to face this problem: a synthetic vaccine based on antigenic peptide epitope selection and a subunit vaccine based on recombinant protein fragments.

Objective: The initial objective of this work is to select the best formulations for a COVID-19 vaccine.

Methodology: For the synthetic vaccine strategy, we initially performed an in silico epitope prediction for the Spike (S) and Nucleoprotein (N) proteins of Sars-Cov-2, combining bioinformatic methodologies. The epitopes selected have been synthesized by a specialized service, analysed by ELISA for their recognition of patient antibodies or T cells, respectively, and formulated in nanoparticles in the presence of adjuvants. For the subunit vaccine, the genes cloned in pET28a were obtained by a custom gene synthesis service, and the proteins were expressed in prokaryotic system and purified. The recombinant proteins were analysed by western blot and then formulated with adjuvants and inoculated in Balb/C mouse. The immunogenicity was determined by ELISA, the T cell response was determined by ELISPOT and plaque reduction neutralization test (PRNT) was used to quantify the presence of neutralizing antibodies.

Results: In its first analysis the epitopes were recognized by confirmed COVID-19 patient's monoclonal antibodies in ELISA assay and the recombinant proteins antigenicity and identity was demonstrated. The preclinical assay results showed promising candidates and formulations. Histopathology data demonstrated that the vaccine formulations were safe for the animals in the dose utilised, whereas subsequent ELISA and ELISPOT assays demonstrated the immunogenicity of our formulations and the ability to elicit a cellular immune response, making them promising candidates for continuous development.

Conclusion: Our initial results show that we have promising candidates for a vaccine development and additional tests have been planned to confirm our preliminary results and advance the product development.

Keywords: vaccine; COVID-19; Sars-CoV-2 